\title{
TERAPIA FONOLÓGICA EM SUJEITOS COM DIFERENTES GRAVIDADES DO DESVIO FONOLÓGICO
}

\author{
Phonological therapy in subjects with different \\ phonological disorder severities
}

\author{
Karina Carlesso Pagliarin ${ }^{(1)}$, Márcia Keske-Soares ${ }^{(2)}$
}

\begin{abstract}
RESUMO
Tema: evolução terapêutica de dois sujeitos com diferentes gravidades do desvio fonológico tratados pelo Empty Set com distinção mínima de traços distintivos. Procedimentos: participaram da pesquisa dois sujeitos, do sexo masculino, com desvio fonológico. S1, com 4:5 anos, apresentava desvio de grau médio-moderado, e S2, com 6:4 anos, com desvio moderado-severo. Ambos foram submetidos ao Empty Set com distinção mínima de traços. Posteriormente, foram comparadas as avaliações fonológicas inicial e final. Resultados: o S1 adquiriu em menor tempo os sons-alvos selecionados, entretanto, ambos os sujeitos apresentaram generalizações para itens não utilizados no tratamento e para outras posições na palavra. Apenas o S2 apresentou generalização dentro de uma classe de sons e para outras classes de sons. Conclusão: o Empty Set com distinção mínima de traços foi eficaz para o tratamento do desvio de grau médio-moderado e moderado-severo, pois ambos apresentaram melhoras na fala.
\end{abstract}

DESCRITORES: Criança; Distúrbios da Fala; Fonoterapia

\section{INTRODUÇÃO}

Estudos têm sido realizados em busca de tratamentos mais eficientes e eficazes para o desvio fonológico ${ }^{1-6}$.

Alguns modelos terapêuticos utilizam a abordagem contrastiva de traços distintivos para a escolha dos sons-alvo, como o modelo de Pares Mínimos ${ }^{7}$, de Oposições Múltiplas ${ }^{8}$, o de Oposições Máximas ${ }^{9}$ e Empty Set ${ }^{10}$.

Esses modelos envolvem pares de duas palavras que diferem em apenas um fonema. Se estes fonemas diferem em poucos traços distintivos formam as Oposições Mínimas, e em vários traços, as Oposições Máximas ${ }^{10}$.

(1) Fonoaudióloga; Professora do Curso de Fonoaudiologia da Universidade Federal de Santa Maria, UFSM, Santa Maria, RS; Mestre em Distúrbios da Comunicação Humana.

(2) Fonoaudióloga; Professora do Curso de Fonoaudiologia e do Mestrado em Distúrbios da Comunicação Humana da Universidade Federal de Santa Maria, UFSM, Santa Maria, RS; Doutora em Linguística Aplicada pela Pontifícia Universidade Católica do Rio Grande do Sul.

Conflito de interesses: inexistente
No Modelo de Oposições Máximas ${ }^{9}$ a seleção dos sons-alvo baseia-se nos erros fonêmicos da criança relativos ao alvo, ou seja, a criança é ensinada a contrastar sons que não são usados apropriadamente, com aqueles que são corretamente usados em seu sistema fonológico, isto é, contrasta um som novo e um som conhecido. Existe ainda, uma variação deste modelo, denominado Empty Set ${ }^{10}$, que da mesma forma que o Modelo de Oposições Máximas é indicado para desvios severos e seleciona alvos com distinção máxima de traços, porém ambos os alvos estão ausentes no sistema fonológico da criança ${ }^{10}$.

Alguns estudos realizados no Brasil ${ }^{5,6,11-13}$ indicam a utilização do Modelo de Oposições Máximas, porém o que se observa é que o tratamento foi a partir do Empty Set, mas as autoras não fazem referência a essa denominação. Porém, neste estudo optou-se pela utilização da nomenclatura original.

O modo como os fonemas de um par mínimo diferem pode ser fundamental na indução de mudanças fonológicas na terapia. Os fonemas de um par mínimo podem diferir em três dimensões linguísticas: número de oposições distintivas 
(minimamente opostos ou maximamente opostos); natureza dos traços distintivos, ou seja, os fonemas podem diferir em traços de classe principal ou classe não principal; e relação com gramática da criança pré-tratamento (o fonema tratado pode estar ausente ou presente no sistema fonológico da criança) ${ }^{10}$.

Tendo isso em vista, neste trabalho, utilizou-se o modelo Empty Set com distinção mínima de traços com o objetivo de comparar a evolução terapêutica de dois sujeitos com diferentes gravidades do desvio fonológico.

\section{APRESENTAÇÃO DO CASO}

Participaram da pesquisa dois sujeitos com queixa de alterações de fala, ambos do sexo masculino. O S1 com 4:5 anos e o S2 com 6:4 anos no início do tratamento.

Primeiramente, os sujeitos foram avaliados através de triagem fonoaudiológica, realizada no Serviço de Atendimento Fonoaudiológico (SAF), em que foi diagnosticado o desvio fonológico, sendo então encaminhados para o Centro de Estudos de Linguagem e Fala (CELF), onde foram submetidos a avaliações mais completas. $O$ diagnóstico de desvio fonológico foi confirmado por meio das avaliações fonoaudiológicas (Avaliação da Linguagem, Avaliação do Sistema Estomatognático, Avaliação de Discriminação Auditiva, Avaliação da Consciência Fonológica, Avaliação da Memória de Trabalho, Avaliação Simplificada do Processamento Auditivo Central, Avaliação Fonológica e Avaliação do Vocabulário - ABFW) e de exames complementares (Otorrinolaringológico, Audiológico e Neurológico). Todas as avaliações encontravam-se dentro dos padrões de normalidade esperados para a faixa etária, com exceção da avaliação fonológica.

Os dados de fala foram obtidos através da Avaliação Fonológica da Criança - AFC ${ }^{14}$ a partir da qual, realizou-se análise contrastiva e o cálculo do Percentual de Consoantes Corretas (PCC) ${ }^{15}$. Com o resultado do PCC o desvio pode ser classificado como desvio médio (86 a 100\%); desvio médiomoderado (66 a 85\%); desvio moderado-severo (51 a $65 \%)$; e desvio severo $(<50 \%)$.

A partir do cálculo do PCC, contatou-se que o S1 apresentava desvio de grau médio-moderado e o S2 apresentava desvio moderado-severo.
Após, as avaliações os sujeitos foram submetidos ao Empty Set ${ }^{10}$, com distinção mínima de traços. As palavras-alvo utilizadas para ambos os sujeitos foram: /Rata/ x /lata/; /Rua/ x /lua/, as quais diferiam em apenas dois traços distintivos de classe não principal.

A estrutura de sessão baseou-se em uma pesquisa ${ }^{16}$, na qual é realizada, inicialmente, a linha de base, ou seja, uma sondagem realizada antes do início da terapia quando cada som não-adquirido é testado, selecionando-se no máximo seis palavras que contenham este fonema e possam ser representadas por figuras, as quais a criança deve nomeá-las.

Após a determinação da linha de base, iniciou-se o tratamento propriamente dito. Foram realizadas cinco sessões de estimulação com os pares selecionados. Na sexta sessão, realizou-se a primeira sondagem, a qual era efetuada da mesma maneira que a linha de base, descrita anteriormente. Se a criança atingisse $50 \%$ de produções corretas, repetiam-se mais cinco sessões com os mesmos pares mínimos em nível de sentença, porém se as produções corretas fossem em percentual inferior a $50 \%$, repetia-se novamente o tratamento em nível de palavra. E, novamente na sexta sessão, realizava-se outra sondagem. Na sondagem, após o ciclo com sentenças, se atingisse $80 \%$ de produções corretas, novos sons-alvo eram determinados. Caso contrário, repetia-se novamente o tratamento em nível de sentença. A sessão terapêutica foi iniciada e terminada com o bombardeio auditivo, além disso, os pais foram orientados quanto ao trabalho de estimulação a ser realizado em casa.

O tratamento das crianças constou de duas sessões semanais de 45 minutos cada, totalizando 30 sessões de terapia, distribuídas da seguinte forma: cinco sessões e uma sondagem. Após o término das 25 sessões foi realizada novamente uma avaliação do sistema fonológico das crianças coletando os dados de fala por meio do mesmo instrumento usado na avaliação inicial. Também foi realizado novamente o cálculo do PCC.

Nas reavaliações (sondagens) o $\mathrm{S} 1$ adquiriu os sons-alvos selecionados, sendo necessário selecionar, novamente, outros sons para tratamento. Nessa ocasião selecionaram-se os alvos $/ r / x / K /$, ambos permaneciam ausentes em seu sistema fonológico. Quanto ao S2, por não ter adquirido os sons-alvo durante as sondagens, foi tratado com os mesmos pares durante as 25 sessões. 
Esta pesquisa foi submetida ao Comitê de Ética em Pesquisa (CEP) sob $n^{\circ} 108 / 05$. Os pais leram e assinaram o Termo de Consentimento Livre e Esclarecido.

Para análise dos dados, foram comparadas as avaliações fonológicas inicial e final.

\section{RESULTADOS}

A Figura 1 refere-se a comparação da avaliação fonológica inicial e final dos segmentos não adquiridos (SNA) no sistema fonológico, com indicação da gravidade inicial e final, dos sons-alvo selecionados e do número de sessões realizadas com cada par.

Observa-se que o $\mathrm{S} 1$ adquiriu em menor tempo os sons-alvo selecionados em relação ao
S2, havendo necessidade de selecionar novos pares. Porém, ambos os sujeitos obtiveram evoluções, principalmente o S2 que apresentava maior número de fonemas ausentes em seu sistema fonológico.

O S1 apresentou generalização para itens não utilizados no tratamento e para outras posições na palavra, pois adquiriu os sons-alvo trabalhados com exceção do fonema $/ r /$.

O S2 adquiriu o fonema /R/ e o generalizou para itens não utilizados no tratamento e para outras posições na palavra. Verifica-se também, que por apresentar maior número de segmentos não adquiridos, devido à gravidade do desvio, obteve generalização para outras classes de sons $(/ \mathrm{g}, / \mathrm{s}, / \mathrm{z} /)$ e dentro de uma classe de sons $(/ / /, / \mathrm{r} /)$.

\begin{tabular}{|c|c|c|c|c|c|c|}
\hline Sujeito & $\begin{array}{c}\text { Gravidade } \\
\text { Inicial }\end{array}$ & AF-I (SNA) & Som-alvo & n Sessões & AF-F (SNA) & $\begin{array}{c}\text { Gravidade } \\
\text { Final }\end{array}$ \\
\hline S1 & DMM & $|\mathrm{I} /,| \mathrm{N} /, / \mathrm{R} /,|\mathrm{r}|$ & $\begin{array}{l}/ R / x / / / \text { - OI } \\
/ r / x / N / \text { - OM }\end{array}$ & $\begin{array}{c}10 \\
15 \\
\text { Total } 25\end{array}$ & $/ r /$ & DM \\
\hline S2 & DMS & $\begin{array}{l}/ \mathrm{k} /, / \mathrm{g} /, / \mathrm{s} /, / \mathrm{z} / \\
/ \mathrm{l} /, / \mathrm{N} /, / \mathrm{R} /, / \mathrm{r} /\end{array}$ & $/ \mathrm{R} / \mathrm{x} / \mathrm{I} / \mathrm{-OI}$ & $\begin{array}{c}25 \\
\text { Total } 25\end{array}$ & $/ \mathrm{k} /, / \mathrm{l} /$ & DM \\
\hline
\end{tabular}

Legenda: DMM: Desvio Médio-Moderado. DMS: Desvio Moderado-Severo. DM: Desvio Médio. OI: onset inicial. OM: onset medial. AF-I. Avaliação Fonológica Inicial. AF-F: Avaliação fonológica Final. SNA: Segmentos Não-Adquiridos

\section{Figura 1 - Comparação dos sujeitos pré e pós-tratamento}

\section{DISCUSSÃO}

De acordo com um estudo ${ }^{10}$, o Empty Set é indicado para o tratamento de desvios severos, entretanto o modelo mostrou-se eficaz para os graus médio-moderado e moderado-severo, pois os sujeitos adquiriram os fonemas ausentes em seus sistemas fonológicos.

A generalização para itens não utilizados no tratamento e para outras posições na palavra foram observadas em ambos os sujeitos. Corroborando em parte os achados de um estudo ${ }^{17}$ que utilizou o modelo Empty Set com distinção mínima de traços e verificou que o sujeito tratado apresentou apenas generalização a itens não utilizados no tratamento.

A distinção mínima de traços favoreceu a ocorrência de generalização para outras classes de sons no S2, esse resultado pode estar relacionado com a complexidade dos fonemas tratados ${ }^{18} \mathrm{O}$ que pode tê-la favorecido. O S1 não apresentava possibilidade de ocorrência para este tipo de gene- ralização uma vez que estavam adquiridos todos os sons possíveis de generalizar. Entretanto, a generalização dentro de uma classe de sons era possível, pois o sujeito não apresentava nenhuma líquida, mas o mesmo não apresentou, havendo necessidade de selecionar esses segmentos para tratamento, no caso $/ \mathrm{r} / \mathrm{x} / \mathrm{K} /$.

Um estudo ${ }^{11}$ realizado com o objetivo de verificar os aspectos estruturais da generalização em quatro sujeitos com desvio fonológico de grau médiomoderado, submetidos ao Modelo Oposições Máximas, constatou a ocorrência dos diferentes tipos de generalização para todos os sujeitos pesquisados.

O S2 obteve generalização dentro de uma classe. Tais achados estão de acordo com uma pesquisa ${ }^{13}$ que utilizou o modelo de oposições com distinção mínima de traços em uma criança com desvio moderado-severo e constatou a presença deste tipo de generalização.

Ambos os sujeitos apresentaram melhoras na fala, visto que no final do tratamento houve um 
aumento do PCC, ou seja, os dois apresentaram desvio de grau médio. Isto vai ao encontro dos achados de diversas pesquisas ${ }^{5,6,10}$ as quais referem que os modelos de abordagem contrastiva favorecem a ocorrência de generalizações o que contribui para rápida evolução terapêutica e consequente alta fonoaudiológica.

\section{CONCLUSÃO}

O Empty Set com distinção mínima de traços foi eficaz para o tratamento dos sujeitos com desvio médio-moderado e moderado-severo, pois ambos apresentaram generalizações e consequentemente, melhoras na fala.

\begin{abstract}
Background: the therapeutic evolution of two subjects with different degree of phonological disorder treated by Empty Set with minimal distinction of distinctive traces. Procedures: the sample was made up by two male subjects with phonological disorder. S1, age 4:5-year old, with mild-moderate phonological disorder, and S2, age 6:4-year old, with moderate-severe phonological disorder. Both were treated by Empty Set with minimal distinction of traces. After treatment, we compared the initial and final phonological evaluation. Results: S1 acquired in less time the selected target sounds, however, both subjects showed generalizations to untreated words and to other word positions. Only S2 showed generalization across and within other sound classes. Conclusion: the Empty Set with minimal distinction of distinctive traces was effective for the treatment of mild-moderate and moderatesevere disorders because both subjects showed improvement as for the speech.
\end{abstract}

KEYWORDS: Child; Speech Disorders; Speech Therapy

\section{REFERÊNCIAS}

1. Crosbie S, Holm A, Dodd B. Intervention for children with severe speech disorder: a comparison of two approaches. Int $\mathrm{J}$ Lang Commun Disord. 2005; 40(4):467-91.

2. Pascoe M, Stackhouse J, Wells B. Phonological therapy within a psycholinguistic framework: promoting change in a child with persisting speech difficulties. Research Report. Int J Lang Commun Disord. 2005; 40(2):189-220.

3. Williams A. A systematic perspective for assessment and intervention: a case study. Adv Speech Lang Pathol. 2006; 8(3):245-56.

4. Gierut JA. Phonological complexity and language learnability. Am J Speech Lang Pathol. 2007; 16(1):6-17.

5. Mota HB, Keske-Soares M, Bagetti T, Ceron MI, Melo Filha MGC. Análise comparativa da eficiência de três diferentes modelos de terapia fonológica. Pró-Fono. 2007; 19(1):67-74.

6. Keske-Soares M, Marini C, Brancalioni AR, Ceron MI, Pagliarin KC. Eficácia da terapia para desvios fonológicos com diferentes modelos terapêuticos. Pró-Fono. 2008; 20(3):153-8.

7. Weiner FF. Treatment of phonological disability using the method of meaningful minimal contrast: two case studies. J Speech Hear Disord. 1981; 46(1):97-103.

8. Williams AL. Multiple oppositions: theoretical foundations for an alternative contrastive intervention approach. Am J Speech Lang Pathol. 2000 Nov; 9(4):282-8.

9. Gierut JA. Maximal opposition approach to phonological treatment. J Speech Hear Disord. 1989; 54(1):9-19.

10. Gierut JA. The conditions and course of clinically induced phonological change. J Speech Lang Hear Res. 1992 Out; 35(5):1049-63.

11. Mota HB, Bagetti T, Keske-Soares M, Pereira LF. A generalização em sujeitos com desvio fonológico médio-moderado tratados pelo modelo de oposições máximas. Rev Soc Bras Fonoaudiol. 2004; 9(2):102-11.

12. Ceron MI, Keske-Soares M. Terapia fonológica: a generalização a itens não utilizados no tratamento (outras palavras). Rev. CEFAC. 2007 out-dez; 9(4):453-60. dx.doi.org/10.1590/ S1516-18462007000400004

13. Ceron MI, Keske-Soares M. Terapia fonológica: a generalização dentro de uma classe de sons e para outras classes de sons. Rev. CEFAC. 2008; 10(3):311-20. dx.doi.org/10.1590/ S1516-18462008000300006 
14. Yavas M, Hernandorena CLM, Lamprecht RR. Avaliação fonológica da criança: reeducação e terapia. Porto Alegre: Artes Médicas; 2001.

15. Shriberg LD, Austin D, Lewis BA, McSweeny JL, Wilson DL. The percentage of consonants correct (PCC) metric: extensions and reliability data. J Speech Lang Hear Res. 1997; 40(4):708-22.

16. Bagetti T, Mota HB, Keske-Soares M. Modelo de oposições máximas modificado: uma proposta de tratamento para o desvio fonológico. Rev Soc Bras Fonoaudiol. 2005 jan-mar; 10(1):36-42.
17. Pagliarin KC, Keske-Soares M, Mota HB. Terapia fonológica em irmãos com diferentes graus de gravidade do desvio fonológico. Rev. CEFAC. 2009; 11(1):20-4. dx.doi.org/10.1590/ S1516-18462009000100004

18. Mota HB. Aquisição segmental do português: um modelo implicacional de complexidade de traços. [tese]. Porto Alegre (RS): Pontifícia Universidade Católica do Rio Grande do Sul; 1996.

RECEBIDO EM: 23/05/2009

ACEITO EM: 26/08/2009

Endereço para correspondência:

Karina Carlesso Pagliarin

Rua Coronel Scherer, 9

São Pedro do Sul - RS

CEP: $97400-000$

E-mail: karinap_fono@yahoo.com.br 